\title{
TEORÍAS GENERALISTAS VERSUS ESPECIFICIDADES DE CLASE, RAZA, EDAD Y ORIENTACIÓN SEXUAL: DIFICULTADES PARA EL FEMINISMO
}

\author{
GENERAL THEORIES VERSUS SPECIFICITIES OF CLASS, RACE, AGE AND SE- \\ XUAL ORIENTATION: DIFFICULTIES FOR FEMINISM
}

Mg. Liudmila Morales Universidad Tecnológica Israel lmorales@uisrael.edu.ec.

Fecha de recepción: 17/03/2015 Fecha de aceptación: 10/04/ 2015

\begin{abstract}
Resumen:
1 presente artículo se acerca a un problema fundamental para las Ciencias Sociales y Humanísticas desde el pasado siglo: las intersecciones del género con otras categorías de identificación y las dificultades que ello plantea para el feminismo como teoría y movimiento emancipatorio. Desentraña tales dificultades a partir del pensamiento de autoras como Pateman, Mouffe, Benhabib, Fraser y Nicholson en tanto clave reflexiva para plantear interrogantes y sobre todo, señalar focos de atención para un entendimiento cabal de opresión social y los sujetos.Palabras clave: Impacto Ambiental, Turismo, Biodiversidad, Índice de calidad de agua, Quebrada.
\end{abstract}

Palabras clave: Teorías feministas, perspectiva interseccional, clase social, raza, etnia. 
Abstract:

This article focuses on a fundamental issue for Social and Humanistic Sciences since the last century: the intersections between gender and other categories of identification and the difficulties it creates for feminism, as a theory and an emancipatory movement. It unravels such difficulties through the thought of authors like Pateman, Mouffe, Benhabib, Fraser and Nicholson, as a reflective key to pose questions and, above all, spotlights for a thorough understanding of social issues and subjects.

Keywords: Feminist theories, intersectional perspective, social class, race, ethnicity. 


\section{Introducción}

¿Cuántas veces hemos deseado ser alguien más? ¿Cuántas veces nos hemos sorprendido queriendo pensar como otro(a), tratando de entender sus pensamientos, intentando imaginar cómo actuaría en determinada situación? Cual frustrante réplica, dice un viejo refrán que nunca se llega a conocer enteramente a una persona, sin importar lo cercana que nos resulte: las sorpresas aguardan siempre tras un recodo del camino.

A tenor del ejemplo, que a primera vista parecería alejarse del saber académico, una pregunta acecha. Si pensar que hablamos por alguien más resulta una especie de atrevimiento, ¿puede una teoría representar a tod@s, como pretenden ciertas corrientes de pensamiento "generalistas"? De afirmarse que sí, daríamos por sentado que luchar contra la opresión de las mujeres es la esencia del feminismo, la base de su programa y la clave de su posible éxito.

Betty Friedan, en La Mística de la Feminidad, creyó que hablaba por las mujeres. Y de cierta forma lo hacía, como voz inicial que al instante halla eco en otras. Pero cuando Friedan condenaba la esclavitud del ama de casa, a salvo de penurias económicas en su caja de cristal de clase media, no plasmaba ni un dos por ciento de las preocupaciones de las norteamericanas que considerarían esas cadenas como cómodas, en comparación con sus propios grilletes de penuria, carencia material, discriminación y negación de oportunidades, por solo citar un ejemplo. Su compatriota bell hooks (2004) se encargó de demostrarlo, en lo que a las mujeres negras respecta. Mucho menos hablaba Friedan por las no norteamericanas o "las condenadas de la tierra", paráfrasis de Frantz Fanon.

Siguiendo la línea de La Mística..., mucho se ha pretendido clamar por la reivindicación de las mujeres, mucho se ha querido encontrar el hilo que las conecte. Son una clase, pero no se reconocen como tal, alegó Simone de Beauvoir (1999). Cabría cuestionarse por qué.

En la bibliografía de corte feminista, dos posturas se delinean. Una, considera las demandas específicas de las mujeres negras, pobres o lesbianas, por ejemplo, como secundarias, ante el imperativo mayor del feminismo: combatir la opresión de las mujeres, en sentido lato. Para las teóricas adscritas a esta vertiente, que pudiéramos llamar generalista, la categoría mujeres contiene la naturaleza de toda la explotación femenina, describiendo así una suerte de subordinación general o mal común. 
Las pensadoras que se rehúsan a una idea generalista de las mujeres, por el contrario, consideran que debe visualizarse a cada mujer como un sujeto concreto e individual, con sus particularidades, porque existen diferentes tipos y grados de opresión. De acuerdo con esta lógica de pensamiento que denominaré particularista ante la ausencia de un término mejor el feminismo necesita revelar las diversas formas de opresión femenina, para ganar en actualidad, representatividad, y poder de convocatoria.

¿Cómo conciliar ambas líneas de pensamiento sin que ello redunde en fracturas dentro de la teoría, ética y práctica política del feminismo (término bajo el cual me permito la libertad de agrupar sus corrientes) y sí en mayor fuerza de este, en tanto movimiento emancipador? Esta deviene una deuda que escritoras como Chantal Mouffe (1995), Nancy Fraser y Linda Nicholson (1990) se han encargado de destacar, frente a producciones teóricas como las de Carole Pateman (1995) cuyas reflexiones sobre la opresión, amén de ciertos ángulos sugerentes, desbordan carácter generalista.

\section{Desarrollo}

Para Pateman (1995) el origen de la subordinación de las mujeres se encuentra en el contrato sexual, paralelo al contrato social que describe John Locke, y "mitad del acuerdo" suprimida. La autora desmembra la teoría contractualista para exponer su subtexto de género, y denuncia el ocultamiento de la parte del trato que implicó la subordinación ulterior de las mujeres a los hombres y el acceso de estos últimos al cuerpo femenino.

A partir del derrocamiento de la ley del padre y su sustitución por una sociedad civil de hermanos, como bien apunta Pateman (1995) en El contrato sexual, el nuevo orden aparentaría ser antipatriarcalista por antonomasia. Más la autora describe al contrato sexual como "una historia de sujeción" cuyas consecuencias ulteriores implican una relación amo-súbdito que desfavorece a las mujeres, porque "la libertad civil es un atributo masculino" y el contrato, "el medio a través del cual el patriarcado moderno se constituye" (Pateman, 1995: p.10-11).

El derecho paterno, entendido como modelo de derecho político, es combatido, mientras que el derecho sexual de los hombres sobre las mujeres prevalece, en tanto es considerado como no político.

De esta disección del contrato, con la consiguiente invisibilización de un segmento, se deriva la división entre las dos esferas: pública y privada, mientras la determinación de relevancia se le adjudica solo a una. Innumerables aristas emanan, a su vez, de la separación de ambas esferas, relacionadas en lo fundamental con una devaluación de los sentimientos y lo que a ellos se asocia, y la ponderación de la racionalidad, característica de lo público y políticamente relevante.

Pateman (1995) sentencia que el confinamiento de las mujeres a la esfera doméstica repercute en una devaluación de las actividades realizadas por ellas, idea que resulta harto cuestionable. 
En primer lugar, debemos aclarar que lo entendido como público o privado y las actividades contenidas en ambos dominios varían de acuerdo con elementos contextuales: geográficos, temporales y culturales, por ejemplo. Otro argumento en contra proviene del debilitamiento limítrofe de las dos esferas, en un momento propicio a la inserción laboral de las mujeres en la sociedad.

No por ello desaparece la opresión, y se sigue desvirtuando el trabajo femenino, por lo que pudiera invertirse el razonamiento de Pateman (1995) y valorar la posibilidad de que se devalúa a la esfera privada en el momento en que se asocia con la mujer, y no viceversa, porque de lo contrario resultaría forzado el hecho de que la desvalorización persiga a la mujer amén de los diferencias contextuales que puedan adquirir las esferas y de la transgresión de sus límites por esta.

El juicio negativo de valor se le adjudicaría entonces a la mujer, y no a la esfera privada. Por otra parte, la idea de que la opresión de las mujeres se manifiesta a través de la relación amo-súbdito, implica la sujeción directa a un hombre y plasma cómo, para Pateman (1995), esta díada se reproduce en las sociedades a través de diferentes tipos de contrato, como el matrimonial, el laboral, y el de prostitución, al que la autora pres- ta especial atención. Pero ¿tiene la relación amo-súbdito suficiente flexibilidad para adherirse a una pluralidad de relaciones de subordinación, marcadas por variables contextuales específicas? ¿Pueden reducirse a esta explicación las múltiples formas en que la subordinación se manifiesta?

Nancy Fraser y Linda Nicholson (1990) tienen mucho que objetar a las ideas de Pateman (1995). En su exploración de las posibilidades de alianza entre feminismo y postmodernismo, las autoras incluirían la lógica de El contrato sexual entre los modos de teorización feminista que se asemejan a las metanarrativas criticadas por los postmodernistas. "They are very large social theories (...) which claim, for example, to identify causes and constitutive features of sexism that operate cross-culturally", conceptualizan Fraser y Nicholson (1990: p. 27).

De ahí que las autoras consideren a dichos modos de teorización "quasi-metanarrati- ves", porque:

they tacitly presuppose some commonly held bay unwarranted and essentialist assumptions about the nature of human beings and the conditions for social life. (...) Such theories, then, share some of the essentialist and ahistorical features of metanarratives: They are insufficiently attentive to historical and cultural diversity, and they falsely universalize features of the theorist's own era, society, culture, class, sexual orientation, and ethnic, or racial group. (Fraser y Nicholson, 1990: p. 27).

Un interesante giro frente a la postura de Pateman (1995) lo provee Chantal Mouffe (1995), con sus reflexiones sobre la ciudadanía y la serie de posiciones de sujeto en que el agente social se constituye, dentro de disímiles formaciones discursivas. Mouffe (1995) afirma que un individuo puede ser dominado en una relación y dominante en otra, por lo que no puede hablarse entonces de una relación monolítica amo-súbdito, y mucho menos de una identidad o conciencia permanente de la subordinación, aglutinante artificial con que no pocas veces se ha pretendido homogeneizar a la categoría mujeres. 
Los planteamientos de Mouffe (1995) son pertinentes en este punto a partir de una interrogante. ¿No es la inclusión de la mujer como agente social activo y ciudadana con plenitud legal de facto uno de los objetivos a posteriori del feminismo y antítesis de varias formas de opresión?

Y digo varias por no absolutizar, pero gran parte del contenido emancipatorio feminista guarda relación con el ámbito político. Basta una mirada a la propia concepción de Pateman (1995) sobre el confinamiento de la mujer a la esfera privada, basado en un entendimiento de la exclusión social como causa y efecto de marginación y opresión de índole diversa. En cuanto al problema rector del presente artículo: la necesidad de visualizar en el feminismo a la mujer como individuo concreto, Mouffe (1995: p. 423) señala que:

sólo cuando descartemos la visión del sujeto como un agente al mismo tiempo racional y transparente para sí mismo, y descartemos también la supuesta unidad y homogeneidad del conjunto de sus posiciones, tendremos posibilidades de teorizar la multiplicidad de las relaciones de subordinación.

Como añadido, figura el imperativo de que no resulten lejanas las demandas feministas a ciertos grupos o sectores que, según las teorías generalistas, deberían verse representados en el término mujer, pero que como bell hooks (2004) demuestra, traen a colación otras variables en juego como la raza, o la orientación sexual. No se trata solo de verse como mujeres, sino como negras, lesbianas, o pobres, por citar, definición que considero previa e indispensable para localizar el origen de ciertos tipos de opresión y, a la postre, devenir feministas, (o no).

El enfoque de Mouffe (1995) permite no solo dirigir la famosa pregunta de Simone de Beauvoir (1999: p. 15) “¿qué es una mujer?” hacia los múltiples contextos en que la categoría se configura, sino arrojar luz sobre el "enigma" de por qué no nos reconocemos como clase. He aquí el peligro de integrar a las mujeres a la categoría clase del marxismo: la eterna ponderación de la semejanza, o igualdad, o elemento en común que venda los ojos para el reconocimiento de que ya pertenecemos a otra clase, la social, y que quizá la influencia cultural de esta, sus prácticas e ideologías asociadas pesen más que cierta conciencia de la opresión sexogenérica. Tal vez esa conciencia de clase explique por qué a veces las burguesas son solidarias con los burgueses y no con las proletarias, hecho que Beauvoir (1999) atribuía a la carencia de una historia en común, de una solidaridad y los medios para unirse.

Es necesario ir más allá en la explicación, profundizar en los motivos por los que alguien se puede sentir más lesbiana que mujer, o más negra que mujer, o más pobre que mujer, aun cuando exista un reconocimiento de la opresión, justo como mujer. Aquí entra en juego la identificación de la causa de esa opresión, que no siempre conduce al género, debido a la superposición de otras variables de identificación, también consideradas como discriminatorias.

La declaración de una esencia común de las mujeres se complica porque, de acuerdo con Mouffe (1995), no es posible adquirir una identidad social permanente.

¿Puede entonces hablarse de las mujeres negras o de las lesbianas o de las pobres o de las jóvenes? La propia autora contesta cuando reconoce que: 


\begin{abstract}
"Esto no significa, sin embargo, que no podamos retener nociones como 'clase trabajadora' 'varones', 'mujeres', 'negros' u otros significantes que se refieren a sujetos colectivos. No obstante, una vez que se ha descartado la existencia de una esencia común, su estatus debe ser concebido en términos de los que Wittgenstein designa como 'parecidos familiares', y su unidad debe ser vista como el resultado de una fijación parcial de identidades mediante la creación de puntos nodales” (Mouffe, 1995: p. 424).
\end{abstract}

Para que la explicación propuesta con anterioridad resulte consecuente y responsable, pasa por tres requisitos:

1. El análisis de cómo presenta a la mujer el feminismo (o el enfoque en cuestión, tal como feminismo de la igualdad, de la diferencia). Implica deslindar desde dónde y para quién hablamos.

2. La visión del individuo como una suma de relaciones contingentes en diversos campos de la vida material, que Mouffe (1995: p. 423) liga a la formación de una "identidad” también contingente y precaria, "dependiente de formas específicas de identificación". Ello conduce a la disyuntiva de cuál forma de identificación prevalece o se prioriza a la hora de exigir determinada reivindicación.

3. El reconocimiento de que la participación política o el activismo social se manifiestan mediante dos motores fundamentales: para alcanzar conquistas o para preservarlas.

Las tres observaciones anteriores facilitan el entendimiento del conflicto entre una teoría generalista de la dominación como la del amo-súbdito y otra que no pierda de vista las manifestaciones específicas de esta dominación en ciertos contextos y relaciones. Asimismo, permite deslindar dónde acaban las diferencias y empiezan los "parecidos familiares" y la creación de puntos nodales, que son, a mi juicio, el punto de partida para una teoría, ética y práctica política feministas más inclusivas.

Sin embargo, esta propuesta encuentra reticencias en quienes, como bien apunta Mouffe (1995, p. 422) consideran irreconciliable hacer a un lado la perspectiva de las mujeres como una identidad social coherente con el hecho de que se unan "como mujeres para formular y perseguir objetivos específicamente feministas".

Se produce así una confusión entre lo que nos une y lo que nos diferencia, motivada por la insistencia en considerar las especificidades como impedimentos para la identificación, y el temor a que sobrepasen en la balanza a aquello que nos asemeja.

Coincido con Chantal Mouffe (1995: p.424), en que la aceptación de que la categoría mujer "no corresponde con ninguna esencia unitaria y unificadora" repercute de manera directa en la estructuración de las luchas políticas del movimiento feminista. En orden de efectuar dicha redirección, urge desmontar desde los cimientos la estructura misma de una teoría feminista, para reconstruirla, a partir de pequeñas piezas.

Como plano para esta reedificación, indica Mouffe (ídem) valorar “¿cómo se convierte la diferencia sexual en una distinción pertinente? y ¿cómo se construyen las relaciones de subordinación a través de tal distinción?”. 
Por supuesto, sin considerar aristas como la raza, la edad, la orientación sexual y la clase, tales preguntas perderían alcance, pues el reiterativo descarte de estas categorías en cuestiones de teoría y política feminista no hace más que negar una verdad sólida como roca.

Esta es: que dichas categorías tienen en el plano social un significado, y comprenden valores correspondientes del orden de lo simbólico. Ello, de un lado, incide en términos de opresión, y del otro, afecta en disímiles modos la conformación de las identidades, su fijación parcial y la creación de puntos nodales.

Y si la ignorancia o negación de estos elementos tiene innegables repercusiones negativas, su reconocimiento transita en sentido opuesto, pero con mayores consecuencias. Así lo asegura Mouffe:

Todo el falso dilema de igualdad versus diferencia se derrumba desde el momento en que ya no tenemos una entidad homogénea mujer enfrentada con otra entidad homogénea varón, sino una multiplicidad de relaciones sociales en las cuales la diferencia sexual está construida siempre de muy diversos modos, y donde la lucha en contra de la subordinación tiene que ser planteada en formas específicas y diferenciales (Mouffe, 1995: p. 424).

La perspectiva del otro concreto deviene una herramienta de utilidad para armonizar estas formas "específicas y diferenciales" con el fortalecimiento de un movimiento que, a no dudar, jamás perderá su pertinencia por la aceptación de que no es posible hablar por las mujeres. A nivel deontológico, el otro concreto y el otro generalizado reflejan las posibilidades de generalizar o particularizar a las mujeres en el feminismo.

\section{Hacia lo concreto}

Seyla Benhabib retoma la controversia Kolberg-Gilligan para contraponer las teorías en torno al otro generalizado y el otro concreto. El primero "nos demanda considerar a todos y cada uno de los individuos como seres racionales, con los mismos derechos y deberes que desearíamos concedernos a nosotros mismos" (Benhabib, 1990: p. 136). Hasta aquí, todo bien, pero como acertadamente enjuicia Benhabib (ídem) "al asumir este punto de vista hacemos abstracción de la individualidad y de la identidad concreta del otro".

Pudiera decirse que dentro de la teoría feminista ha prevalecido en el plano ético el punto de vista del otro generalizado, con las mismas sanas intenciones con que, en el marco de la Revolución Francesa, se pretendió que "Libertad, Igualdad y Fraternidad" fuese un lema que abarcara a todos y no solo a los propietarios blancos de la pujante burguesía.

En oposición, el punto de vista del otro concreto impele a considerar a cada quien "como un individuo con una historia, una identidad y una constitución afectivo-emocional concretas" (ídem). Por ende, de lo que se hace abstracción es de lo común. Para el feminismo, establecer como norma ética esta perspectiva, sería dar por sentado o desestimar lo que nos une como mujeres (aunque algunos pondrían en duda la validez misma de la categoría, una vez que se ha hecho abstracción de lo común) e ir en busca de las 
especificidades, con la idea rectora de que "nuestras diferencias se complementan en lugar de excluirse mutuamente" (Benhabib, 1990: p. 136).

Un área de permanente indagación devendría el mecanismo de complemento, ante el reto que plantea la exclusión, por su aparente cercanía con las diferencias. Sería bueno imaginar que, contempladas como otro concreto, personas como Bell Hoks (2004) no hubieran experimentado el abismo que las separaba de las feministas blancas y sentido una especie de rechazo de estas hacia las demandas puntuales de las negras.

Desatender el punto de vista del otro concreto "lleva a incoherencias epistémicas en las teorías morales universalistas" (Benhabib, 1990: p. 139) porque "no se puede llevar a cabo una prueba coherente de universabilidad, pues carecemos de la información epistémica suficiente para juzgar si mi situación moral es 'semejante' a la tuya o si no lo es" (Benhabib, 1990: p.143).

He ahí el error de las teorías feministas construidas sobre un principio de universalidad: intentar equiparar la situación moral de un ama de casa o profesional burguesa con la de una anciana pobre, negra y lesbiana, porque en teoría ambas son "súbditas". Simularía la cuadratura del círculo el imbricar las reivindicaciones de ambos extremos, por lo que Benhabib inquiere si

Dado que son nuestras identidades en tanto que otros concretos lo que hace que nos distingamos unos de otros en razón de las diferencias culturales, de género, de clase y de raza, así como las capacidades psíquicas y naturales, una teoría moral restringida al punto de vista del otro concreto, ¿podría no ser racista, sexista, relativista cultural y discriminatoria? Además, se puede argüir que sin (...) el otro generalizado no es pensable una teoría política de la justicia que sea adecuada para las modernas sociedades complejas (Benhabib, 1990: p.143).

La respuesta había sido dada antes, cuando la propia Benhabib (1990) presentaba a las diferencias como puente y no como medio de exclusión, idea que no queda totalmente clara en El otro generalizado y el otro concreto... pero que interpreto con la metáfora de descomponer la estructura ideológica y moral, para volverla a edificar, como mencionaba antes.

De cualquier manera, si hubiera cabida para una teoría generalista en las sociedades del capitalismo tardío (y el emergente socialismo del siglo XXI) sería erigida a partir de piezas contextualizadas y concretas, los parecidos similares y puntos nodales de Wittgenstein y Mouffe (1995), porque cada vez se hace más difícil y moralmente hipócrita mantener la ilusión de un otro generalizado y paradójico.

El "universalismo interactivo" al que se refiere Benhabib (1990: p.144) proveería el primer eslabón de la cadena, en tanto "reconoce que el otro generalizado también es un otro concreto"; pero no puede quedarse en el reconocimiento el camino hacia el otro concreto, si este reconocimiento es formal y figura solo como una nota a pie de página. Parte de la visibilización del otro como individuo cruzado por innumerables tangentes es darle voz, porque la misma Benhabib (1990) alerta sobre el peligro de hablar por los otros, inquietud con la que dio inicio este artículo. 
Fraser y Nicholson (1990: p.35) divergerían con Benhabib (1990) sobre la proposición de cualquier tipo de universalismo, porque "a postmodern feminist theory would be nonuniversalist. When it focus became cross cultural or trans epochal, its mode of attention would be comparativist rather than universalist". Convergen aquí los presupuestos teóricos del postmodernismo.

Benhabib (1990: p.147) misma pondría en tela de juicio al universalismo cuando admite que "los discursos sobre las necesidades y motivos se despliegan en este espacio creado por lo común y lo único, la socialización compartida de un modo general, y la contingencia de las historias individuales", al esbozar los puntos de encuentro y desencuentro del feminismo con la mujer, como individuo particular.

Otro desliz cometido por Benhabib (1990: p.148) la lleva a argüir cómo, "para entender y combatir la opresión de las mujeres ya no basta con exigir únicamente la emancipación política y económica de estas" y cuando se creería que iba a añadir manifestaciones particulares relacionadas con la discriminación racial, por orientación sexual, o la subordinación de muchísimas ancianas al resto de los miembros del hogar, muestra un punto coincidente con Pateman (1995).

"También es necesario poner en cuestión las relaciones psicosexuales de las esferas doméstica y privada dentro de las que se desarrolla la vida de las mujeres, y a través de las cuales se reproduce la identidad de género" (ídem).

Aquí Benhabib (1990) no distingue que la reproducción de dicha identidad trasciende los límites entre esferas. Limita así el punto de vista del otro concreto, que en todo caso, "es un concepto crítico que designa los límites ideológicos del discurso universalista" e incluye "lo no pensado, lo no visto y lo no oído de esas teorías" (Benhabib, 1990: p.144). No hay nada de "no visto" o "no oído" en su pretensión, si ya se ha establecido que asociar la opresión solo con los marcos de la esfera doméstica tuerce los senderos hacia la justicia, en especial si se entiende a esta última como una "justice of multiplicities", cual Fraser y Nicholson (1990) señalan, retomando a Lyotard.

Las autoras agregarían también cómo la vuelta de Benhabib hacia la esfera doméstica replica la monocausalidad de teorizar continuamente "in terms of a putatively unitary, primary, culturally universal type of activity associated to women, generally and activity conceived as domestic and located in the family" (Fraser y Nicholson, 1990: p.29). 


\section{Conclusiones}

- Para un feminismo más inclusivo, a las referidas posibilidades del otro concreto en el orden del deber ser, Mouffe (1995: p. 435) añadiría la necesidad de descartar el proyecto de una política "específica y estrictamente feminista (...) diseñada para la persecución de los intereses de las mujeres como mujeres, sino más bien como la persecución de las metas y aspiraciones feministas dentro del contexto de una más amplia articulación de demandas". Pero la autora, o bien se contradice, o bien presenta la anterior como una alternativa a las formas precarias de identificación respecto a la categoría mujeres "que provean la base para una identidad feminista y una lucha feminista" (ídem: p.434).

- Ambas alternativas parecen más acertadas que la concepción generalista de las mujeres, pero la primera guarda mayor coherencia con los ideales del otro concreto, y del feminismo como movimiento emancipador por excelencia. Estoy de acuerdo con Mouffe (1995) en que la inserción de las demandas propiamente feministas en un marco social más extenso conlleva a transformar las diferentes prácticas, discursos y relaciones sociales en que la categoría mujer se instituye en intríngulis con la subordinación.

- La propuesta de Fraser y Nicholson guarda, de igual manera, mayor relación con la primera variante de Mouffe, porque, como base para una práctica política, la teoría feminista postmoderna "would replace unitary notions of woman and feminine gender identity with plural and complexly constructed conceptions of social identity, treating gender as one relevant strand among others, attending also to class, race, ethnicity, age, and sexual orientation" (Fraser y Nicholson, 1990: p.35).

- Me adscribo a quienes no comparten la visión del género como único e impoluto pilar a la hora de analizar las diversas formas de opresión femenina. Encuentro difícil hablar de emancipación de las mujeres cuando el imperativo mayor es subsistir en un mundo que en efecto les niega oportunidades, pero más si permanecen por fuera de las estructuras raciales de la "supremacía blanca", carecen de medios económicos, o no reproducen los valores de la heterosexualidad normativa. Partir de ahí conlleva a estructurar, en consecuencia, una lucha plural que contemple posibles soluciones, así en plural también, y que no ensaye supuestas panaceas universales.

- Fraser y Nicholson señalan de forma acertadísima la ventaja que implica tal visión, traducida en utilidad para la práctica política feminista contemporánea. A esta práctica, la describen como surcada por la premisa de que "while some women share some common interests and face some common enemies, such commonalities are by no means universal; rather, they are interlaced with differences, even with conflicts. This, then, is a practice made up of a patchwork of overlapping alliances, not one circumscribable by an essential definition" (Fraser y Nicholson, 1990: p.35).

- Ergo, un sujeto contingente y fluctuante por diversas posiciones necesita identificarse, en un sentido no excluyente de sus múltiples dimensiones sociales. Una mujer X, cuyo única causa de opresión no es ser la 
súbdita de un amo Y, debe encontrar en el feminismo, o en la práctica de los feminismos -ampliación de Fraser y Nicholson (1990)- la salida para el círculo vicioso que estar al fondo de la injusta pirámide social significa. Ello solo es posible si identificarse como mujer no redunda en una identificación forzada de un sujeto limitado, que encuentra nuevas contingencias donde parecía abrirse una puerta. 


\section{Bibliografía}

Beauvoir, S. (1999). El segundo Sexo. Buenos Aires: Editorial Sudamericana.

Benhabib, S. (1990). El otro generalizado y el otro concreto: la controversia Kohlberg-Gilligan y la teoría feminista. En Benhabib, Seyla y Cornella, Drucilla. Teoría feminista y teoría crítica. Ensayos sobre la política de género en las sociedades del capitalismo tardío. Valencia: Edicions Alfonso El Magnánim.

Benhabib, S. y Cornella, D. (1990). Teo- ría feminista y teoría crítica. Ensayos sobre la política de género en las sociedades del capitalismo tardío. Valencia: Edicions Alfonso El Magnánim.

Fraser, N. y Nicholson, L. (1990). “Social Criticism without Philosophy: An encounter between Feminism and Postmodernism”. En Nicholson, Linda (ed.) Feminism/Postmodernism. New York: Routledge.

Hooks, B. (2004). "Mujeres negras. Dar forma a la teoría feminista". En Otras inapropiables. Feminismos desde las fronteras. bell hooks, Avtar Brah, Chela Sandoval, Gloria Anzaldúa, Madrid: Traficantes de sueños.

Mouffe, Ch. (1995). "Feminismo, ciudadanía y política democrática radica”l. En Grompone, Romeo (ed.) Adrianzén, Alberto (comp); Cotler, Julio (comp) y López, Sinesio (comp) Instituciones políticas y sociedad. Lima: IEP Ediciones.

Nicholson, L. (ed.) (1990). Feminism/ Postmodernism. New York: Routledge.

Pateman, C. (1995) El contrato sexual. México: ANTHROPOS-UA 
